\title{
Efficient CPU and GPU implementations of multicenter integrals over long-range operators using Cartesian Gaussian functions
}

\author{
Alexander Humeniuk ${ }^{\mathrm{a}, \mathrm{b}, *}$, William J. Glover ${ }^{\mathrm{a}, \mathrm{b}, \mathrm{c}, *}$ \\ ${ }^{a}$ NYU Shanghai, 1555 Century Avenue, Shanghai 200122, China \\ ${ }^{b}$ NYU-ECNU Center for Computational Chemistry at NYU Shanghai, 3663 Zhongshan Road North, Shanghai 200062, China \\ ${ }^{c}$ Department of Chemistry, New York University, New York, New York 10003, USA
}

\begin{abstract}
We present a library for evaluating multicenter integrals over polarization operators of the form $x^{m_{x}} y^{m_{y}} z^{m_{z}} r^{-k} C(r)$ using Cartesian Gaussian basis functions. $m_{x}, m_{y}, m_{z} \geq 0, k>2$ are integers, while the cutoff function, $C(r)=\left(1-e^{-\alpha r^{2}}\right)^{q}$, with $\alpha \in \mathbb{R}_{+}$and certain integer values of $q$ ensures the existence of the integrals. The formulation developed by P. Schwerdtfeger and H. Silberbach [Phys. Rev. A 37, 2834 (1988)] is implemented in an efficient and stable way taking into account a recent fix in one of the equations. A cheap upper bound is presented that allows negligible integrals to be prescreened. The correctness of the analytical integrals was verified by numerical integration. The library provides separate codes for serial CPU and parallel GPU architectures and can be wrapped into a python module.
\end{abstract}

Keywords: polarization integrals; polarizable embedding; Gaussian basis sets; core polarization potentials; one-electron integrals; long-range interaction; multipole expansion

\section{PROGRAM SUMMARY}

Program Title: polarization_integrals

CPC Library link to program files: (to be added by Technical Editor)

Developer's repository link: github.com/humeniuka/polarization_integrals Code Ocean capsule: (to be added by Technical Editor)

Licensing provisions: MIT

Programming language: $\mathrm{C}++$, CUDA, python

Nature of problem(approx. 50-250 words):

Evaluating multicenter integrals of long-range operators between

Cartesian Gaussian basis functions

Solution method(approx. 50-250 words):

Corrected version of Schwerdtfeger's formulation from Refs. [1,2,3]

\section{References}

[1] P. Schwerdtfeger, H. Silberbach, Multicenter integrals over long-range operators using Cartesian Gaussian functions, Phys. Rev. A 37, 2834 (1988).

[2] P. Schwerdtfeger, H. Silberbach, Erratum, Phys. Rev. A 37, 2834 (1988),

[3] P. Schwerdtfeger, H. Silberbach, Erratum, Phys. Rev. A 103 069901(2021).

\section{Introduction}

Multicenter integrals over long-range operators of the type

$$
\mathrm{O}(\boldsymbol{r})=x^{m_{x}} y^{m_{y}} z^{m_{z}} r^{-k}
$$

with $k>2$ and $m_{x, y, z} \in \mathbb{N}_{0}$ arise in various contexts of quantum chemistry. For instance, the operator representing the polarization energy, $\hat{H}_{\text {pol }}=-\frac{1}{2} \hat{\boldsymbol{f}} \boldsymbol{A} \hat{\boldsymbol{f}}$, which depends quadratically on

\footnotetext{
${ }^{*}$ Corresponding authors
}

E-mail addresses: alexander.humeniuk@gmail.com,william.glover@nyu.edu the electric field operator $\hat{f}$ and some effective polarizability tensor $\boldsymbol{A}$, is of this type. For a single atom having a dipole polarizability $\alpha$ interacting with a single electron, the polarization potential takes the form $V_{\mathrm{pol}}(r)=-\frac{1}{2} \alpha r^{-4}$. Integrals of such polarization operators are found for example in core polarization potentials (CPP) that are used to model core-valence electron correlation in atoms and molecules $[1,2]$ and the direct reaction field method (DRF) [3, 4] which is a polarizable embedding scheme for quantum mechanics/molecular mechanics (QM/MM) simulations.[5] DRF requires matrix elements of polarization operators between basis functions on different atomic centers. Historically these integrals were approximated by a Taylor expansion of the electric fields around an arbitrary reference position [6] to avoid the exact evaluation. However, since the choice of the expansion center is not a differentiable operation, analytic gradients of the polarization energy with respect to nuclear coordinates were not easily accessible. Quadrupole and higher-order polarizability corrections also require matrix element for a range of different values of $m_{x, y, z}$ and $k$.[7] In general, terms of the form in Eqn. (1) appear in multipole expansions of the electrostatic potential.

P. Schwerdtfeger and H. Silberbach were the first to succeed in solving integrals of the operator (1) between Cartesian Gaussian basis functions in full generality.[8, 9] Their derivation distinguishes different subcases which are solved separately. As a side node we would like to mention that Smit has presented an alternative formulation,[10] which makes use of the McMurchie-Davidson recurrence relations.[11] While this recursive approach is expected to be more efficient than Schwerdtfeger's, we are not aware of any published implementation.

This article provides a library written in $\mathrm{C}++$ for evaluating 
multicenter integrals of polarization operators between Cartesian Gaussians. CUDA kernels that take advantage of the acceleration provided by graphical processing units (GPU) are also included. For convenience the integral routines can be exported as a python module. This library thus complements existing open source quantum chemistry integral packages for other one- and two-electron operators, such as GEN1INT and libint.[12, 13]

Outline: The reader is assumed to be familiar with the notation in the original articles by Schwerdtfeger and Silberbach.[8, 9, 14] Primed equations refer to the first article (Ref. [8]). After defining the quantities of interest, some space is devoted to the subcase 2.2 , which contained a mistake that was fixed only recently.[14] Then the efficient and numerically stable calculation of certain auxiliary functions is explained in detail. In section 4.1 an upper bound on the polarization integrals based on the Cauchy-Schwarz inequality is given that allows negligible integrals to be efficiently prescreened. Finally, section 5 explains how to use the library. Expressions for nuclear gradients of the polarization integrals are provided in the appendix.

\section{Polarization Integrals}

The polarization integrals are defined as the matrix elements of the operator $x^{m_{x}} y^{m_{y}} z^{m_{z}} r^{-k}$

$$
I_{i 0 j}=\left\langle\phi_{i}\left|x^{m_{x}} y^{m_{y}} z^{m_{z}} r^{-k}\left(1-e^{-\alpha r^{2}}\right)^{q}\right| \phi_{j}\right\rangle
$$

between unnormalized Cartesian Gaussian-type orbitals (CGTO)

$$
\phi_{i}(x, y, z)=\left(x-x_{i}\right)^{n_{x i}}\left(y-y_{i}\right)^{n_{y i}}\left(z-z_{i}\right)^{n_{z i}} \exp \left(-\beta_{i}\left(\boldsymbol{r}-\boldsymbol{r}_{i}\right)^{2}\right)
$$

where $\boldsymbol{r}_{i}=\left(x_{i}, y_{i}, z_{i}\right)$ is the center of the basis function, $n_{x i}, n_{y i}, n_{z i} \in \mathbb{N}_{0}$ define its angular momentum and $\beta_{i} \in \mathbb{R}_{+}$is the orbital exponent parameter. The cutoff function, $\left(1-e^{-\alpha r^{2}}\right)^{q}$, with $\alpha \in \mathbb{R}_{+}$ensures that the integrals exist for $k>2$ provided the power of the cutoff function $q$ satisfies

$$
q \geq \kappa(k)-\kappa\left(m_{x}\right)-\kappa\left(m_{y}\right)-\kappa\left(m_{z}\right)-1
$$

where

$$
\kappa(n)=\left\{\begin{array}{ll}
n / 2 & \text { if } n \text { is even } \\
(n+1) / 2 & \text { is } n \text { is odd }
\end{array} .\right.
$$

For simplicity the polarization operator is centered at the origin, but integrals for any other location can be obtained by shifting the coordinate system so that its origin coincides with the center of the operator.

\section{Implementation}

The implementation of the integral routines is based on the formulation in Refs. [8, 9]. For the sake of brevity, no attempt is made here to summarize the intricate derivations of all the different subcases. However, we noticed a small mistake in the case for $k=2 j+1$ and $s-j<0$ (case 2, subcase 2), which has been corrected only recently in an erratum [14]. Since the argument is rather subtle, the problem will be explained below at some length.

\subsection{Subcase 2.2: $k=2 j+1$ and $s-j<0$}

At the end of section III in Ref. [8] the claim is made that $\ldots$, all one center contributions of matrix elements of operator $r^{-2 j-1}$ vanish if $j-s-1 \geq 0$.

This statement is wrong as will be shown below and casts doubt on the validity of Eqn. (40') in combination with $u_{1^{\prime}}^{s}(j, a, b)$.

To illustrate this, we calculate the integral of the operator $r^{-3}$ ( $j=1$ ) between two s-orbitals with exponent $\beta=1$ positioned at the origin $(s=0$ so that $j-s-1=0)$. According to the statement above it should vanish, but it clearly does not. For simplicity, the cutoff function $\left(1-\exp \left(-r^{2}\right)\right)^{2}$ is used $(\alpha=1$, $q=2$ ). The resulting integral is one-dimensional:

$$
\begin{aligned}
I_{000} & =\int d^{3} r \phi_{i}(\boldsymbol{r}) r^{-3} \phi_{j}(\boldsymbol{r})\left(1-e^{-r^{2}}\right)^{2} \\
& =\int_{0}^{\infty} d r\left(4 \pi r^{2}\right) e^{-r^{2}} \frac{1}{r^{3}} e^{-r^{2}}\left(1-e^{-r^{2}}\right)^{2} \\
& =4 \pi \int_{0}^{\infty} \frac{1}{r}\left(e^{-2 r^{2}}-2 e^{-3 r^{2}}+e^{-4 r^{2}}\right) \\
& =\pi \log \left(\frac{81}{64}\right) \\
& \approx 0.74005
\end{aligned}
$$

In line 3 of the above equation, summation and integration cannot be exchanged and the integral has been evaluated analytically using Mathematica [15]. Numerical integration on a grid gives the same value to six decimal places.

For comparison, we now evaluate the same integral using Eqn. (40') in the limit $b \rightarrow 0\left(a_{\mu}=2+\mu\right)$ :

$$
\begin{aligned}
I_{000} & =\Gamma^{-1}\left(\frac{3}{2}\right) \pi^{\frac{3}{2}} 2 \sum_{\mu=0}^{2}\left(\begin{array}{l}
2 \\
\mu
\end{array}\right)(-1)^{\mu}(2+\mu)^{0} \sum_{v=0}^{1}\left(\begin{array}{l}
1 \\
v
\end{array}\right)(-1)^{v} H(1-v, 0) \\
& =0
\end{aligned}
$$

$I_{000}$ vanishes independently of $H(1-v, 0)$, because there is no dependence on $\mu$ and $\sum_{\mu=0}^{q}\left(\begin{array}{l}q \\ \mu\end{array}\right)(-1)^{\mu}=0$. This is in accordance with the statement in the article but it contradicts the previous calculations. Therefore Eqn. (40') must be wrong for the case $k=2 j+1$ and $s-j<0$.

The error in subcase $k=2 j+1$ with $s-j<0$ can be traced back to the statement

Since this term is independent of $a_{\mu}$, we get ...

just above the Eqn. (48'). This statement is not correct since $\epsilon$ in fact does depend on $a_{\mu}$; however, this dependence got lost when replacing the limit $\eta \rightarrow \infty$ with a different limit $\epsilon \rightarrow 1$ in Eqn. (31'). Consequently Eqn. (48') has a limit different from 0 . To derive the correct limit, we have to go back to Eqn. (31') and repeat the derivation for case $2 \mathrm{~b}$, while keeping track of $a_{\mu}$ in the integration limits. 
The starting point is

$$
\begin{aligned}
J_{2}=\lim _{\eta \rightarrow \infty} & \sum_{\mu=0}^{q}\left(\begin{array}{l}
q \\
\mu
\end{array}\right)(-1)^{\mu}\left(\frac{a_{\mu}}{b^{2}}\right)^{s+3 / 2} \\
& \times \int_{0}^{\eta} d t\left(\frac{b^{2}}{a_{\mu}}-\frac{b^{2}}{a_{\mu}+t}\right)^{s+3 / 2} t^{j-s-2} e^{b^{2} /\left(a_{\mu}+t\right)}
\end{aligned}
$$

Making the substitution

$$
\begin{aligned}
z(t) & =\frac{b^{2}}{a_{\mu}}-\frac{b^{2}}{a_{\mu}+t} \\
t(z) & =b^{2}\left(\frac{b^{2}}{a_{\mu}}-z\right)^{-1}-a_{\mu}=a_{\mu}\left(\frac{b^{2}}{a_{\mu}}-z\right)^{-1} z \\
d t & =b^{2}\left(\frac{b^{2}}{a_{\mu}}-z\right)^{-2} d z
\end{aligned}
$$

the integral transforms into

$$
\begin{aligned}
J_{2}=\lim _{\eta \rightarrow \infty} \sum_{\mu=0}^{q}\left(\begin{array}{l}
q \\
\mu
\end{array}\right) & (-1)^{\mu} a_{\mu}^{j-s-1}\left(\frac{a_{\mu}}{b^{2}}\right)^{s+1 / 2} e^{b^{2} / a_{\mu}} \\
& \times \int_{0}^{\frac{b^{2}}{a_{\mu}}-\frac{b^{2}}{a_{\mu}+\eta}} d z\left(\frac{b^{2}}{a_{\mu}}-z\right)^{s-j} z^{j-1 / 2} e^{-z}
\end{aligned}
$$

The important point is that the limit is written as

$$
\lim _{\eta \rightarrow \infty} \int_{0}^{\frac{b^{2}}{a_{\mu}}-\frac{b^{2}}{a_{\mu}+\eta}} \ldots
$$

and not as

$$
\lim _{\epsilon \rightarrow 1} \int_{0}^{\left(\frac{b^{2}}{a_{\mu}}\right) \epsilon} \ldots
$$

Defining $p=j-s>0$ and another substitution

$$
\begin{aligned}
x^{2} & =\frac{a_{\mu}}{b^{2}} z \\
z & =\frac{b^{2}}{a_{\mu}} x^{2} \\
d z & =2 \frac{b^{2}}{a_{\mu}} x d x
\end{aligned}
$$

turn the integral into

$$
\begin{aligned}
& J_{2}= \\
& 2 \lim _{\eta \rightarrow \infty} \sum_{\mu=0}^{q}\left(\begin{array}{l}
q \\
\mu
\end{array}\right)(-1)^{\mu} a_{\mu}^{p-1} e^{b^{2} / a_{\mu}} \int_{0}^{\sqrt{\frac{\eta}{a_{\mu}+\eta}}} d x \frac{x^{2 j}}{\left(1-x^{2}\right)^{p}} e^{-\left(b^{2} / a_{\mu}\right) x^{2}} .
\end{aligned}
$$

The recursion relations in Eqns. (35'), (36') and (37a,b') are still valid, however, Eqn. (37c') needs revision. To obtain $H(1, a)$ we have to solve the integral $J_{2}$ for $j=0$ and $p=1$.

Setting temporarily $a=\frac{b^{2}}{a_{\mu}}$ and using the trick

$$
\frac{1}{1-x_{1}^{2}}=\int_{0}^{\infty} d x_{2} e^{-\left(1-x_{1}^{2}\right) x_{2}}
$$

the relevant integral becomes

$$
\begin{aligned}
H_{\eta}(1, a) & =\int_{0}^{\sqrt{\frac{\eta}{a_{\mu}+\eta}}} \frac{1}{1-x^{2}} e^{-a x^{2}} \\
& =e^{-a} \int_{0}^{\sqrt{\frac{\eta}{a_{\mu}+\eta}}} d x_{1} \int_{0}^{\infty} d x_{2} e^{\left(1-x_{1}^{2}\right)\left(a-x_{2}\right)} .
\end{aligned}
$$

The integral over $\mathbb{R}_{+}$is split into $[0, a](a, \infty)$

$$
\begin{aligned}
& H_{\eta}(1, a)=e^{-a} e^{\left(1-x_{1}^{2}\right)\left(a-x_{2}\right)} \\
& \quad \times\left[\int_{0}^{\sqrt{\frac{\eta}{a_{\mu}+\eta}}} d x_{1} \int_{0}^{a} d x_{2}+\int_{0}^{\sqrt{\frac{\eta}{a_{\mu}+\eta}}} d x_{1} \int_{a}^{\infty} d x_{2}\right] .
\end{aligned}
$$

The first integral exists in the limit $\eta \rightarrow \infty$ and we get

$$
H(1, a)=e^{-a} \int_{0}^{1} d x_{1} \int_{0}^{a} d x_{2} e^{\left(1-x_{1}^{2}\right)\left(a-x_{2}\right)}=\sqrt{\pi} e^{-a} m(\sqrt{a}) .
$$

This is identical to Eqn. (37c') except for a factor of $\sqrt{\pi} / 2$ which is due to a more conventional definition of the error function.

The real problem rests with the second integral, which after substituting $u=a-x_{2}$ and integrating over $u$ and then $x_{1}$ gives

$$
\begin{gathered}
e^{-a} \int_{0}^{\sqrt{\frac{\eta}{a_{\mu}+\eta}}} d x_{1} \int_{a}^{\infty} d x_{2} e^{\left(1-x_{1}^{2}\right)\left(a-x_{2}\right)}=e^{-a} \int_{0}^{\sqrt{\frac{\eta}{a_{\mu}+\eta}}} d x_{1} \frac{1}{1-x_{1}^{2}} \\
=e^{-b^{2} / a_{\mu}} \tanh ^{-1}\left(\sqrt{\frac{\eta}{a_{\mu}+\eta}}\right)
\end{gathered}
$$

This expression diverges in the limit $\eta \rightarrow \infty$. However, in Eqn. (18) (setting $p=1$ ) it occurs never alone but only in combination with other diverging integrals that are summed with different signs so that they cancel:

$$
\begin{aligned}
J_{2}(p=0, j= & 1)=2 \sum_{\mu=0}^{q}\left(\begin{array}{l}
q \\
\mu
\end{array}\right)(-1)^{\mu} e^{b^{2} / a_{\mu}} H(1, a) \\
& +2 \lim _{\eta \rightarrow \infty} \sum_{\mu=0}^{q}\left(\begin{array}{l}
q \\
\mu
\end{array}\right)(-1)^{\mu} \tanh ^{-1}\left(\sqrt{\frac{\eta}{a_{\mu}+\eta}}\right) .
\end{aligned}
$$

What is the $\eta \rightarrow \infty$ limit in the second line of the previous equation? For sufficiently large $\eta\left(\eta \gg a_{\mu}\right)$, we can approximate

$$
\sqrt{\frac{\eta}{a_{\mu}+\eta}} \approx 1-\frac{a_{\mu}}{2 \eta}
$$

With the help of the identity $\tanh ^{-1}(x)=$ $\frac{1}{2}(\log (1+x)-\log (1-x))$, we get

$$
\begin{aligned}
\tanh ^{-1}\left(\sqrt{\frac{\eta}{a_{\mu}+\eta}}\right) & \stackrel{\eta \gg a_{\mu}}{\longrightarrow} \tanh ^{-1}\left(1-\frac{a_{\mu}}{2 \eta}\right) \\
& =\frac{1}{2}\left[\log \left(2-\frac{a_{\mu}}{2 \eta}\right)-\log \left(a_{\mu}\right)+\log (2 \eta)\right] .
\end{aligned}
$$


Putting this expression back into the second line of Eqn. (24), all limits can be determined:

$$
\begin{aligned}
2 & \lim _{\eta \rightarrow \infty} \sum_{\mu=0}^{q}\left(\begin{array}{l}
q \\
\mu
\end{array}\right)(-1)^{\mu} \tanh ^{-1}\left(\sqrt{\frac{\eta}{a_{\mu}+\eta}}\right) \\
= & \sum_{\mu=0}^{q}\left(\begin{array}{l}
q \\
\mu
\end{array}\right)(-1)^{\mu} \lim _{\eta \rightarrow \infty} \log \left(2-\frac{a_{\mu}}{2 \eta}\right)+\lim _{\eta \rightarrow \infty} \log (2 \eta) \sum_{\mu=0}^{q}\left(\begin{array}{l}
q \\
\mu
\end{array}\right)(-1)^{\mu} \\
& -\sum_{\mu=0}^{q}\left(\begin{array}{l}
q \\
\mu
\end{array}\right)(-1)^{\mu} \log \left(a_{\mu}\right) .
\end{aligned}
$$

Using the binomial theorem $0=(1-1)^{q}=\sum_{\mu=0}^{q}\left(\begin{array}{c}q \\ \mu\end{array}\right)(-1)^{\mu}$, it is clear that the first two terms in Eqn. (27) vanish. Therefore, Eqn. (24) can finally be written as

$$
\begin{aligned}
& J_{2}(p=0, j=1)= \\
& 2 \sum_{\mu=0}^{q}\left(\begin{array}{l}
q \\
\mu
\end{array}\right)(-1)^{\mu} e^{b^{2} / a_{\mu}}\left(H(1, a)-\frac{1}{2} \log \left(a_{\mu}\right) e^{-b^{2} / a_{\mu}}\right) .
\end{aligned}
$$

Now it is very easy to fix the equations for case $2 b$ in Ref. [8]. In summary:

- In view of the usual definition of the error function, $\operatorname{erf}(x)=\frac{2}{\sqrt{\pi}} \int_{0}^{x} d t e^{-t^{2}}$, Eqn. (37b') should better be written as

$$
H(0, x)=\frac{\sqrt{\pi}}{2} x^{-1 / 2} \operatorname{erf}(\sqrt{x}),
$$

- Eqn. (37c') has to be replaced with

$$
H\left(1, \frac{b^{2}}{a_{\mu}}\right)=e^{-b^{2} / a_{\mu}}\left(\sqrt{\pi} m\left(b / \sqrt{a_{\mu}}\right)-\frac{1}{2} \log \left(a_{\mu}\right)\right)
$$

- and Eqn. (48') has to be replaced with

$$
\begin{aligned}
& 2 \lim _{\eta \rightarrow \infty} \sum_{\mu=0}^{q}\left(\begin{array}{l}
q \\
\mu
\end{array}\right)(-1)^{\mu} \tanh ^{-1}\left(\sqrt{\frac{\eta}{a_{\mu}+\eta}}\right)= \\
& -\sum_{\mu=0}^{q}\left(\begin{array}{c}
q \\
\mu
\end{array}\right)(-1)^{\mu} \log \left(a_{\mu}\right) .
\end{aligned}
$$

The corrected analytical expressions for the subcase 2.2 were checked against numerical integrals computed using Becke's multicenter integration scheme $[16,17]$. The spherical grids on each center consisted of 60 radial and 194 angular points each. For the radial distribution of the integration points the Slater radius of hydrogen was chosen. The results are shown in Table 1.

We do not believe the error in Ref. [8] affects any other previously published results, since the problematic case does not occur for the type of integrals needed by standard CPPs.[1, 2] However, subcase 2.2 of the above integrals will be needed for quadrupole polarizability corrections [7].

\subsection{Efficient evaluation of certain special functions}

The integrals in Refs. [8, 9, 14] depend on certain functions $d(p+1 / 2, x)$ and $\gamma(p+1 / 2, x)$, for which Schwerdtfeger et al. give closed-form expressions. For numerical evaluation, however, it is more efficient to define the functions in terms of recursion relations, which follow from partial integrations.

\subsubsection{The function $d(p+1 / 2, x)$ for $p \in \mathbb{Z}$}

The integral is defined as

$$
d_{p}=d(p+1 / 2, x)=\int_{0}^{x} d \omega \omega^{p-1 / 2} e^{\omega} .
$$

For $p=0$ the integral can be expressed with the help of the Dawson function $\left(D_{+}(x)=e^{-x^{2}} \int_{0}^{x} d t e^{t^{2}}\right)$ as

$$
\begin{aligned}
d_{0} & =d(1 / 2, x)=\int_{0}^{x} d \omega \frac{e^{\omega}}{\sqrt{\omega}} \stackrel{\omega=u^{2}}{=} \int_{0}^{\sqrt{x}}(2 u d u) \frac{1}{u} e^{u^{2}} \\
& =2 e^{x}\left(e^{-x} \int_{0}^{\sqrt{x}} d u e^{u^{2}}\right) \\
& =2 e^{x} D_{+}(\sqrt{x}) .
\end{aligned}
$$

For positive integers $p$, the integrals can be obtained iteratively starting from $d_{0}$. The recursion relation comes from the following partial integration $\left(\int f g^{\prime}=f g-\int f^{\prime} g\right)$ :

$$
\begin{aligned}
d_{p} & =\int_{0}^{x} d \omega \omega^{p-1 / 2} e^{\omega}=\int_{0}^{x} d \omega \omega^{p-1 / 2} \frac{d}{d \omega} e^{\omega} \\
& =\left[\omega^{p-1 / 2} e^{\omega}\right]_{0}^{x}-\left(p-\frac{1}{2}\right) \int_{0}^{x} d \omega \omega^{(p-1)-1 / 2} e^{\omega} \\
& =x^{p-1 / 2} e^{x}-\left(p-\frac{1}{2}\right) d_{p-1}
\end{aligned}
$$

For negative $-p$, the recursion relation can also be derived via partial integration $\left(\int f^{\prime} g=f g-\int f g^{\prime}\right)$ :

$$
\begin{aligned}
d_{-p}= & \lim _{\eta \rightarrow 0} \int_{\eta}^{x} d \omega \omega^{-p-1 / 2} e^{\omega} \\
\stackrel{p . i .}{=} & \lim _{\eta \rightarrow 0}\left[\frac{1}{-p+\frac{1}{2}} \omega^{-p+1 / 2}\right]_{\eta}^{x}-\frac{1}{-p+\frac{1}{2}} \int_{0}^{x} d \omega \omega^{-p+1 / 2} e^{\omega} \\
= & \frac{1}{-p+\frac{1}{2}} x^{-p+1 / 2} e^{x}-\lim _{\eta \rightarrow 0} \frac{1}{-p+\frac{1}{2}} \eta^{-p+1 / 2} e^{\eta} \\
& -\frac{1}{-p+\frac{1}{2}} d_{-(p-1)} .
\end{aligned}
$$

Strictly speaking, this integral does not exist because the second term diverges in the limit $\eta \rightarrow 0$. However, the integral never occurs alone but only in certain combinations with similar integrals so that the divergent terms cancel. Keeping this restriction in mind, one can simply delete the divergent term, so that the recursion relation becomes

$$
d_{-p}=\frac{1}{-p+\frac{1}{2}}\left(x^{-p+1 / 2} e^{x}-d_{-(p-1)}\right) .
$$

After substituting $p+1$ for $p$ in Eqns. (34) and (36) the final prescription for calculating $d_{p}=d(p+1 / 2, x)$ becomes

$$
\begin{aligned}
d_{0} & =2 e^{x} D_{+}(\sqrt{x}) \\
d_{p+1} & =x^{p+1 / 2} e^{x}-\left(p+\frac{1}{2}\right) d_{p} \\
d_{-(p+1)} & =-\frac{1}{p+\frac{1}{2}}\left(x^{-(p+1 / 2)} e^{x}-d_{-p}\right) .
\end{aligned}
$$

Note that in these expressions, $p$ is always a non-negative integer. 


\begin{tabular}{lccccrrr}
\hline Case & $\mathrm{k}$ & $m_{3}$ & $z_{1}$ & $z_{2}$ & original $^{a}$ & corrected $^{b}$ & numerical $^{c}$ \\
\hline 1 & 4 & 0 & -1.0 & 0.5 & +18.8461676 & +18.8461676 & +18.8461681 \\
2.1 & 3 & 1 & -1.0 & 0.5 & -0.2983447 & -0.2983447 & -0.2983446 \\
2.2 & 3 & 0 & -1.0 & 0.5 & +0.1452474 & +4.7975293 & +4.7975284 \\
2.2 & 3 & 0 & 0.0 & 0.0 & 0.0 & +16.2380595 & +16.2380596 \\
\hline
\end{tabular}

Table 1: Validation of analytic integrals of Eqn. (2) for the operator $z^{m_{3}} r^{-k}$ between two s-orbitals with exponents $\beta_{1}=\beta_{2}=1$ placed at $\boldsymbol{r}_{1}=\left(0,0, z_{1}\right)$ and $\boldsymbol{r}_{2}=\left(0,0, z_{2}\right)$ (see Ref. [8] for the definition of the different cases). The $z$-coordinates of the orbitals are varied so that $b=\left|\beta_{1} \boldsymbol{r}_{1}+\beta_{2} \boldsymbol{r}_{2}\right|>0$ or $b=0$. A cutoff function with $\alpha=50.0$ and $q=2$ was used. ${ }^{a}$ Using analytic expressions from Refs. [8, 9]. ${ }^{b}$ Using corrected analytic expressions from this work and Ref. [14]. ${ }^{c}$ Numerical evaluation of Eqn. (2).

\subsubsection{The function $\gamma(p+1 / 2, x)$ for $p \in \mathbb{N}_{0}$}

This integral is defined as

$$
g_{p}=\gamma(p+1 / 2, x)=\int_{0}^{x} d z z^{p-1 / 2} e^{-z}
$$

and can also be expressed with the Gamma function, $\Gamma(p+1 / 2)$, and the regularized lower incomplete Gamma function, $P(p+$ $1 / 2, x)$ :

$$
\gamma(p+1 / 2, x)=\Gamma(p+1 / 2) P(p+1 / 2, x) .
$$

For $p=0$, the integral is related to the error function (the definition of the error function in Eqn. (5') of Ref. [8] lacks the usual prefactor of $\left.\frac{2}{\sqrt{\pi}}\right)$ :

$$
\begin{aligned}
g_{0} & =\int_{0}^{x} d z \frac{1}{\sqrt{z}} e^{-z} \stackrel{z=u^{2}, d z=2 u d u}{=} \int_{0}^{\sqrt{x}} d u 2 u \frac{1}{u} e^{-u^{2}} \\
& =2 \int_{0}^{\sqrt{x}} d u e^{-u^{2}}=2 \frac{\sqrt{p i}}{2} \operatorname{erf}(\sqrt{x}) \\
& =\sqrt{\pi} \operatorname{erf}(\sqrt{x}) .
\end{aligned}
$$

An iterative procedure for computing $\gamma(p+1 / 2, x)$ for $p>0$ follows from partial integration:

$$
\begin{aligned}
g_{p} & =\int_{0}^{x} d z z^{p-1 / 2} e^{-z}=\int_{0}^{x} d z z^{p-1 / 2} \frac{d}{d z}\left(-e^{-z}\right) \\
& =\left[z^{p-1 / 2}\left(-e^{-z}\right)\right]_{0}^{x}-\int_{0}^{x} d z \frac{d}{d z}\left(z^{p-1 / 2}\right)\left(-e^{-z}\right) \\
& =-x^{p-1 / 2} e^{-x}+\left(p-\frac{1}{2}\right) \int_{0}^{x} d z z^{(p-1)-1 / 2} e^{-z} \\
& =-x^{p-1 / 2} e^{-x}+\left(p-\frac{1}{2}\right) g_{p-1} .
\end{aligned}
$$

Replacing $p$ with $p+1$ gives the iteration prescription for computing $g_{p}=\gamma(p+1 / 2, x)$ for $p=0,1,2, \ldots$ :

$$
\begin{aligned}
g_{0} & =\sqrt{\pi} \operatorname{erf}(\sqrt{x}) \\
g_{p+1} & =-x^{p+1 / 2} e^{-x}+\left(p+\frac{1}{2}\right) g_{p} .
\end{aligned}
$$

\subsubsection{The Dawson-error hybrid function $m(x)$}

The function $m(x)$ is defined as the integral

$$
m(x)=\int_{0}^{x} d t e^{t^{2}} \operatorname{erf}(t) .
$$

There seem to be no common libraries that provide this function.

In the following, the Taylor expansion of $m(x)$ around an arbitrary point is worked out. The Taylor expansion requires the derivatives

$$
m^{(n)}\left(x_{0}\right)=\left.\frac{d^{n} m}{d x^{n}}\right|_{x=x_{0}}
$$

evaluated at the expansion point $x_{0}$. Using the definition of the error function, $\operatorname{erf}(x)=\frac{2}{\sqrt{\pi}} \int_{0}^{x} d t e^{-t^{2}}$, the first derivatives are found to be

$$
\begin{aligned}
& m^{(1)}=e^{x^{2}} \operatorname{erf}(x) \\
& m^{(2)}=2 x e^{x^{2}} \operatorname{erf}(x)+e^{x^{2}}\left(\frac{2}{\sqrt{\pi}} e^{-x^{2}}\right)=2 x m^{(1)}+\frac{2}{\sqrt{\pi}} \\
& m^{(3)}=2 m^{(1)}+2 x m^{(2)} \\
& m^{(4)}=4 m^{(2)}+2 x m^{(3)} \\
& m^{(5)}=6 m^{(3)}+2 x m^{(4)}
\end{aligned}
$$

This sequence suggests the following induction hypothesis

$$
m^{(n)}=2(n-2) m^{(n-2)}+2 x m^{(n-1)} \quad \text { for } n>2
$$

which is proven easily

$$
\begin{aligned}
m^{(n+1)} & =2(n-2) m^{(n-1)}+2 m^{(n-1)}+2 x m^{(n)} \\
& =2((n+1)-2) m^{((n+1)-2)}+2 x m^{((n+1)-1)} .
\end{aligned}
$$

The Taylor series of $m$ around a point $x_{0}$ at which its value $m\left(x_{0}\right)$ is known is given by

$$
m(x)=\sum_{n=0}^{\infty} \frac{\left(x-x_{0}\right)^{n}}{n !} m^{(n)}\left(x_{0}\right)
$$

with

$$
\begin{aligned}
& m^{(0)}=m\left(x_{0}\right) \\
& m^{(1)}=e^{x_{0}^{2}} \operatorname{erf}\left(x_{0}\right) \\
& m^{(2)}=2 x_{0} m^{(1)}+2 \pi^{-\frac{1}{2}} \\
& m^{(n)}=2(n-2) m^{(n-2)}+2 x_{0} m^{(n-1)} \quad \text { for } n>2 .
\end{aligned}
$$


For the special point $x_{0}=0$, a more compact form can be derived. Since

$$
m^{(0)}(0)=0, \quad m^{(1)}(0)=0, \quad m^{(2)}(0)=2 \pi^{-\frac{1}{2}}
$$

and

$$
m^{(n)}(0)=2(n-2) m^{(n-2)}(0) \quad \text { for } n>2
$$

it follows that the odd derivatives all vanish at the origin,

$$
m^{(2 k+1)}(0)=0 \quad \text { for } k=0,1,2, \ldots
$$

Applying Eqn. (62) repeatedly to the even derivatives yields

$$
\begin{aligned}
m^{(2 k)} & =\prod_{i=2}^{k} 2(2 i-2) 2 \pi^{-\frac{1}{2}}=2 \pi^{-\frac{1}{2}} 2^{2(k-1)} \prod_{i=2}^{k}(i-1) \\
& =\pi^{-\frac{1}{2}} 2^{2 k-1}(k-1) ! \quad \text { for } k>1 .
\end{aligned}
$$

The Taylor expansion around $x=0$ is thus

$$
m(x)=\frac{x^{2}}{\sqrt{\pi}}\left(1+\sum_{k=2}^{\infty} x^{2(k-1)} 2^{2 k-1} \frac{(k-1) !}{(2 k) !}\right) .
$$

For large $x$, one can combine the asymptotic expansion of the Dawson function given in Eqn. (68') of Ref. [8] with the asymptotic expansion of $m(x)$ in Eqn. (74') to show that $m(x)$ is approximately

$$
m(x)=e^{x^{2}} \operatorname{erf}(x) D_{+}(x) \text { for large } x .
$$

For $x \geq x_{L}=6$ this approximation is almost exact. In this case we use the Faddeeva package to evaluate the error and Dawson functions.[18]

For $x<x_{L}$, the interval $\left[0, x_{L}\right]$ is divided into segments at $N$ points $x_{0}=0<x_{1}<x_{2}<\ldots<x_{N-1}<x_{L}$. The initial values needed for starting the iteration according to Eqn. (60) are precalculated and hard-coded as two arrays (see table 2 below):

$$
\begin{aligned}
& m_{i}^{(0)}=m\left(x_{i}\right) \\
& m_{i}^{(1)}=e^{x_{i}^{2}} \operatorname{erf}\left(x_{i}\right) \quad i=0,1, \ldots, N-1 .
\end{aligned}
$$

If $x_{i} \leq x<x_{i+1}$, a truncated Taylor expansion (with $n \leq n_{\max }=$ 20) around $x_{i}$ is used to evaluate $m\left(x_{i}\right)$. Figure 1 compares the piecewise Taylor expansion with the implementation in Mathematica.

For large $x$, the exponential factor $\exp \left(x^{2}\right)$ in Eqn. (66) causes overflows. Therefore it is more stable to compute the function $m^{*}(x)=\exp \left(-x^{2}\right) m(x)$, since the factor $\exp \left(-x^{2}\right)$ can be cancelled elsewhere.

\subsubsection{Binomial Coefficients}

The motif

$$
\sum_{k=0}^{n}\left(\begin{array}{l}
n \\
k
\end{array}\right) x^{n-k} \cdots
$$

occurs frequently in the nested for-loops. The binomial coefficients satisfy the relation

$$
\left(\begin{array}{c}
n \\
k+1
\end{array}\right)=\frac{n !}{(k+1) !(n-k-1) !}=\frac{n !}{(k+1) k ! \frac{(n-k) !}{n-k}}=\frac{n-k}{k+1}\left(\begin{array}{l}
n \\
k
\end{array}\right) \text {. }
$$

\begin{tabular}{ccc}
\hline$x_{0}$ & $m^{(0)}\left(x_{0}\right)$ & $m^{(1)}\left(x_{0}\right)$ \\
\hline 0 & 0 & 0 \\
$1 / 2$ & 0.1536288593561963 & 0.6683350724948156 \\
1 & 0.8153925207417932 & 2.290698252303238 \\
$3 / 2$ & 3.230822260808236 & 9.166150419904208 \\
2 & 15.47726567802307 & 54.34275435683373 \\
$5 / 2$ & 114.4689886643230 & 517.8020183042809 \\
3 & 1443.356844958733 & 8102.904926424203 \\
$7 / 2$ & 31266.84178403753 & 208981.1335760574 \\
4 & $1.149399290121673 \times 10^{6}$ & $8.886110383508415 \times 10^{6}$ \\
$9 / 2$ & $7.107314602194292 \times 10^{7}$ & $6.229644420759607 \times 10^{8}$ \\
5 & $7.354153746369724 \times 10^{9}$ & $7.200489933727517 \times 10^{10}$ \\
$11 / 2$ & $1.269164846178781 \times 10^{12}$ & $1.372170497746480 \times 10^{13}$ \\
\hline
\end{tabular}

Table 2: Centers, $x_{0}$, and initial values, $m\left(x_{0}\right)$ and $m^{\prime}\left(x_{0}\right)$, for local Taylor expansions in the interval $[0,6)$. The Dawsonerror hybrid function at the expansion points was calculated as $m(x)=\frac{x^{2}{ }_{2} F_{2}\left(1,1 ; \frac{3}{2}, 2 ; x^{2}\right)}{\sqrt{\pi}}$ using Mathematica with a precision of 16 digits.

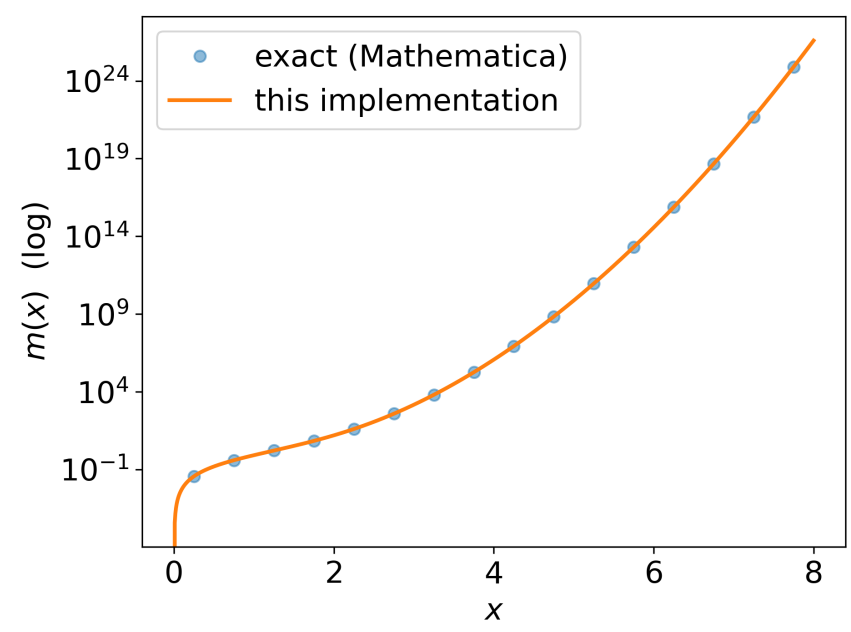

Figure 1: Logarithmic plot of the Dawson-error hybrid function $m(x)$. The relative errors at the test points (blue dots), which include locations half-way between the expansion points, are always smaller than $10^{-10}$.

Replacing $k$ by $k-1$ gives the iteration prescription for downward iteration from $\left(\begin{array}{l}n \\ n\end{array}\right)=1$

$$
\left(\begin{array}{c}
n \\
k-1
\end{array}\right)=\frac{k}{n-k+1}\left(\begin{array}{l}
n \\
k
\end{array}\right) \text {. }
$$

The summand in Eqn. (69),

$$
B_{n, k}=\left(\begin{array}{l}
n \\
k
\end{array}\right) x^{n-k}
$$

can be calculated iteratively in reverse order $(k=n, n-1, \ldots, 0)$ using

$$
\begin{aligned}
B_{n, n} & =1 \\
B_{n, k-1} & =\frac{x k}{n-k+1} B_{n, k} .
\end{aligned}
$$


Terms in the sums of type

$$
\sum_{k=0}^{n}\left(\begin{array}{l}
n \\
k
\end{array}\right) x^{k} \ldots=\sum_{k=0}^{n} A_{n, k} \ldots
$$

are calculated by upward iteration,

$$
\begin{aligned}
A_{n, k+1} & =\left(\begin{array}{c}
n \\
k+1
\end{array}\right) x^{k+1}=\frac{n ! x^{k+1}}{(n-k-1) !(k+1) !} \\
& =\frac{n-k}{(n-k) !} \frac{n !}{(k+1) k !} x^{k} x=x \frac{n-k}{k+1}\left(\begin{array}{l}
n \\
k
\end{array}\right) x^{k} \\
& =x \frac{n-k}{k+1} A_{n, k},
\end{aligned}
$$

starting from

$$
A_{n, 0}=1
$$

\subsection{The limit $b=\left|\beta_{1} \boldsymbol{r}_{1}+\beta_{2} \boldsymbol{r}_{2}\right| \rightarrow 0$}

Ref. [8] only covers the case $b=0$ when $\boldsymbol{r}_{1}=\boldsymbol{r}_{2}=0$. However, as the erratum mentions, [9] $\beta_{1} \boldsymbol{r}_{1}=-\beta_{2} \boldsymbol{r}_{2}$ also leads to $b=0$, i.e. when the centroid of the two Gaussian basis functions happens to coincide with the operator center. As $b$ approaches 0 , the working equations become unstable, since $b$ appears in the denominator. There is no physical problem with this point, one just has to rearrange the equations, as we show below.

\subsubsection{The case $k=2 j$ with $j=2,3, \ldots$ (case 1$)$}

The problematic expression is (below Eqn. (40') in Ref. [8]):

$$
\begin{aligned}
& u_{0}^{s}(j, a, b)= \\
& b^{-2 s+2 j-3} \sum_{v=0}^{j-1}\left(\begin{array}{c}
j-1 \\
v
\end{array}\right)(-1)^{v}\left(\frac{a}{b^{2}}\right)^{v} d\left(s-j+v+1+\frac{1}{2}, \frac{b^{2}}{a}\right) .
\end{aligned}
$$

To cancel the diverging terms, the factor $x^{p+1 / 2}$ is pulled out of the function $d(p+1 / 2, x)$, so that the limit $x \rightarrow 0$ exists for the modified function,

$$
\tilde{d}\left(p+\frac{1}{2}, x\right)=x^{-p-1 / 2} d\left(p+\frac{1}{2}, x\right) .
$$

Using this expression to replace $d$ with $\tilde{d}$ in Eqn. (78) gives

$$
u_{0}^{s}(j, a, b)=a^{-s+j-3 / 2} \sum_{v=0}^{j-1}\left(\begin{array}{c}
j-1 \\
v
\end{array}\right)(-1)^{v} \tilde{d}\left(s-j+v+1+\frac{1}{2}, \frac{b^{2}}{a}\right) .
$$

For $p \geq 0$ one can find a Taylor expansion of $\tilde{d}(p+1 / 2, x)$ around $x=0$. We start from Eqn. (32) and differentiate with respect to $x$ :

$$
d^{\prime}(p+1 / 2, x)=x^{p-1 / 2} e^{x}=x^{p-1 / 2} \sum_{k=0}^{\infty} \frac{x^{k}}{k !} .
$$

Integrating the Taylor expansion formally gives

$$
d\left(p+\frac{1}{2}, x\right)=x^{p+1 / 2} \sum_{k=0}^{\infty} \frac{x^{k}}{k !\left(p+k+\frac{1}{2}\right)} .
$$

For $-p<0$, we rewrite the recursion relations in Eqn. (39) in terms of the $\tilde{d}_{p}$ 's:

$$
\begin{aligned}
\tilde{d}_{-(p+1)} & =x^{p+1 / 2} d_{-(p+1)} \\
& =x^{p+1 / 2}\left(-\frac{1}{p+1 / 2}\right)\left(x^{-p-1 / 2} e^{x}-x^{-p+1 / 2} \tilde{d}_{-p}\right) \\
& =-\frac{1}{p+\frac{1}{2}}\left(e^{x}-x \tilde{d}_{-p}\right) .
\end{aligned}
$$

In summary, for $\frac{b^{2}}{a}<10^{-2}$, Eqn. (78) is replaced by

$$
u_{0}^{s}(j, a, b)=a^{-s+j-3 / 2} \sum_{v=0}^{j-1}\left(\begin{array}{c}
j-1 \\
v
\end{array}\right)(-1)^{v} \tilde{d}_{s-j+v+1}
$$

with

$$
\begin{aligned}
\tilde{d}_{p} & =\sum_{k=0}^{\infty} \frac{x^{k}}{k !\left(p+k+\frac{1}{2}\right)} \\
\tilde{d}_{-(p+1)} & =-\frac{1}{p+\frac{1}{2}}\left(e^{x}-x \tilde{d}_{-p}\right)
\end{aligned}
$$

and $x=\frac{b^{2}}{a}$. The Taylor series is truncated at $k=20$.

\subsubsection{The case $k=2 j+1$ with $j=1,2, \ldots$ assuming $s-j \geq 0$} (case 2, subcase 1)

In this case the offending integral is (second expression below Eqn. (40') in Ref. [8]):

$$
\begin{gathered}
u_{1}^{s}(j, a, b)=a^{j-s-1} \exp \left(\frac{b^{2}}{a}\right) \sum_{v=0}^{s-j}\left(\begin{array}{c}
s-j \\
v
\end{array}\right)(-1)^{v}\left(\frac{a}{b^{2}}\right)^{j+v+1 / 2} \\
\times \gamma\left(j+v+\frac{1}{2}, \frac{b^{2}}{a}\right) .
\end{gathered}
$$

The $\gamma$ function, whose Taylor expansion around $x=0$ we are interested in, is related to the $d$ function:

$$
\begin{aligned}
\gamma(a, x) & =\int_{0}^{x} d t e^{-t} t^{a-1} \stackrel{\omega=-t}{=}(-1)^{a} \int_{0}^{-x} d \omega e^{\omega} \omega^{a-1} \\
& =(-1)^{a} d(a,-x) .
\end{aligned}
$$

The Taylor expansion of $d$ has already been determined in Eqn. (82), so that one finds

$$
\begin{aligned}
\gamma\left(p+\frac{1}{2}, x\right) & =(-1)^{p+1 / 2} d\left(p+\frac{1}{2},-x\right) \\
& =(-1)^{p+1 / 2}(-x)^{p+1 / 2} \sum_{k=0}^{\infty} \frac{(-x)^{k}}{k !\left(p+k+\frac{1}{2}\right)} \\
& =x^{p+1 / 2} \tilde{d}\left(p+\frac{1}{2},-x\right) .
\end{aligned}
$$

Substituting this into Eqn. (87) leads to an expression which is well-behaved in the limit $b \rightarrow 0$ :

$$
u_{1}^{s}(j, a, b)=a^{j-s-1} \exp \left(\frac{b^{2}}{a}\right) \sum_{v=0}^{s-j}\left(\begin{array}{c}
s-j \\
v
\end{array}\right)(-1)^{v} \tilde{d}\left(j+v+\frac{1}{2},-\frac{b^{2}}{a}\right)
$$


3.3.3. The case $k=2 j+1$ with $j=1,2, \ldots$ assuming $s-j<0$ (case 2, subcase 2)

For the last case, the corresponding expression,

$$
u_{1^{\prime}}^{s}(j, a, b)=2 a^{j-s-1} \exp \left(\frac{b^{2}}{a}\right) \sum_{v=0}^{j}\left(\begin{array}{l}
j \\
v
\end{array}\right)(-1)^{v} H\left(j-s-v, \frac{b^{2}}{a}\right)
$$

has a well-defined limit for $b \rightarrow 0$, provided the limits $\lim _{x \rightarrow 0} H(p, x)$ exist. $H(-p, x)$ and $H(0, x)$ are problematic, since they contain terms of the form $x^{-i}$ with $i \geq \frac{1}{2}$, which diverge in the limit $x \rightarrow 0$. For negative $-p$ we use Eqn. (89) to rewrite Eqn. (39') in [8] as

$$
\begin{aligned}
H(-p, x) & =\frac{1}{2} \sum_{v=0}^{p}\left(\begin{array}{l}
p \\
v
\end{array}\right)(-1)^{v} x^{-v-1 / 2} \gamma\left(v+\frac{1}{2}, x\right) \\
& =\frac{1}{2} \sum_{v=0}^{p}\left(\begin{array}{l}
p \\
v
\end{array}\right)(-1)^{v} \tilde{d}\left(v+\frac{1}{2},-x\right) .
\end{aligned}
$$

For $p \geq 0, H(p, x)$ is defined recursively. The only problematic term is the initial value,

$$
H(0, x)=\frac{\sqrt{\pi}}{2} x^{-\frac{1}{2}} \operatorname{erf}(\sqrt{x})=x^{-1 / 2} \int_{0}^{x} d t e^{-t^{2}},
$$

because of the factor $x^{-1 / 2}$. Let us define the function $e(x)=$ $\int_{0}^{x} d t e^{-t^{2}}$ and find its Taylor expansion around $x=0$. The derivatives $e^{(i)}=\frac{d^{i} e}{d x^{i}}$ are:

$$
\begin{aligned}
e^{(0)}(x) & =\int_{0}^{x} d t e^{-t^{2}} \\
e^{(1)}(x) & =e^{-x^{2}} \\
\vdots & \\
e^{(n)}(x) & =-2\left((n-2) e^{(n-2)}(x)+x e^{(n-1)}(x)\right) \text { for } n>1
\end{aligned}
$$

The recursion relation for the derivatives is proven easily:

$$
\begin{aligned}
e^{(n+1)} & =-2\left((n-2) e^{(n-1)}+e^{(n-1)}+x e^{(n)}\right) \\
& =-2\left(((n+1)-2) e^{((n+1)-2)}+x e^{((n+1)-1)}\right)
\end{aligned}
$$

At $x=0$ the derivatives take on the values $e_{n}:=\frac{d^{n} e}{d x^{n}}(0)$

$$
\begin{aligned}
e_{0} & =0 \\
e_{1} & =1 \\
\vdots & \\
e_{n} & =-2(n-2) e^{(n-2)} \text { for } n>1 .
\end{aligned}
$$

It follows that all even derivatives vanish at $x=0$,

$$
e_{2 k}=0 \quad k=0,1, \ldots,
$$

while the odd derivatives are

$$
e_{2 k+1}=-2(2 k-1) e_{2(k-1)+1}
$$

or

$$
e_{2 k+1}=(-2)^{k}(2 k-1) ! !
$$

as can be proven by induction

$$
\begin{aligned}
e_{2(k+1)+1} & =(-2)^{k+1}(2(k+1)-1) ! !=-2(2 k+1)(-2)^{k}(2 k-1) ! ! \\
& =-2(2(k+1)-1) e_{2((k+1)-1)+1} .
\end{aligned}
$$

The Taylor expansion of $e(x)$ around $x=0$ is thus

$$
\begin{aligned}
e(x) & =x\left(1+\sum_{k=1}^{\infty} \frac{x^{2 k}}{(2 k+1) !} e_{2 k+1}\right) \\
& =x\left(1+\sum_{k=1}^{\infty}\left(-2 x^{2}\right)^{k} \frac{(2 k-1) ! !}{(2 k+1) !}\right) \\
& =x\left(1+\sum_{k=1}^{\infty} \frac{\left(-2 x^{2}\right)^{k}}{(2 k+1)(2 k) ! !}\right)
\end{aligned}
$$

For small $x<1.5, H(0, x)$ is therefore computed as

$$
H(0, x)=x^{-1 / 2} e(\sqrt{x})=1+\sum_{k=1}^{\infty} \frac{(-2 x)^{k}}{(2 k+1)(2 k) ! !} .
$$

The Taylor series is truncated at $k=20$.

\section{Upper Bounds for Polarization Integrals}

Our interest in polarization integrals stems from the direct reaction field method.[3] DRF divides the system into an active region (solute, QM), where electrons are treated using quantum mechanics, and an inert region (solvent, MM) of polarizable atoms that model the polarization response of the solvent to the solute density. Assuming that the basis set for the QM part contains $N_{\mathrm{AO}}$ atom-centered orbitals and is surrounded by $N_{\text {pol }}$ polarizable sites, the number of unique polarization integrals scales as $O\left(N_{\mathrm{AO}}^{2} N_{\mathrm{pol}}\right)$. Although the number of combinations of basis functions increases quadratically, asymptotically the number of non-zero one-electron matrix elements scales roughly linearly with the size of the system. This is because atomic orbitals decay exponentially, so that two basis functions have to be relatively close for their product to give a non-negligible contribution to the charge density. Therefore, local operators, like Eq. (2), are sparse in the atomic orbital basis. To take advantage of this sparsity, we seek a simple upper bound to the integrals that allows us to efficiently estimate their magnitude and prescreen out negligible integrals.

\subsection{Cauchy-Schwarz bounds}

A rigorous upper bound follows from the Cauchy-Schwarz (CS) inequality for the scalar product between two vectors: $\langle\boldsymbol{u}, \boldsymbol{v}\rangle \leq|\boldsymbol{u} \| \boldsymbol{v}|$. Let $\mu(\boldsymbol{r})$ and $v(\boldsymbol{r})$ be atomic orbitals centered on different atoms and $O(\boldsymbol{r})=x^{m_{x}} y^{m_{y}} z^{m_{z}} r^{-k}\left(1-e^{-a r^{2}}\right)^{q}$ be the polarization operator with the cutoff function included. The polarization integral can be expressed as a scalar product between the product $\mu(\boldsymbol{r}) \nu(\boldsymbol{r})$ and $O(\boldsymbol{r})$.

$$
\begin{aligned}
& \int d^{3} r \mu(\boldsymbol{r}) O(\boldsymbol{r}) v(\boldsymbol{r})=\langle\mu \nu, O\rangle \stackrel{\text { C.S. }}{\leq}|\mu \nu \| O| \\
= & \left(\int d^{3} r O(\boldsymbol{r})^{2}\right)^{1 / 2}\left(\int d^{3} r \mu(\boldsymbol{r})^{2} v(\boldsymbol{r})^{2}\right)^{1 / 2} .
\end{aligned}
$$


The bounds allow us to neglect pairs of localized basis functions in different regions of the QM part, whose densities overlap very little. In this case, all polarization integrals for that pair of basis functions are screened, no matter where the polarizable atom is placed. As a result, it should be noted that the bounds are not especially tight since they do not take advantage of the decay of the polarization integral with the distance between the polarizable site and the basis functions. However, the CS bounds serve their purpose as a simple and efficient means to prescreen polarization integrals with knowledge of only the atomic orbitals' centers, contraction coefficients, and exponents.

Eqn. (109) is not yet suitable for practical applications: For one thing we wish to screen shells based on the total angular momentum $l=n_{x}+n_{y}+n_{z}$ rather than on the individual Cartesian powers $n_{x}, n_{y}$ and $n_{z}$. Also, it should be computationally cheap to evaluate the upper bound, so numerical integration is out of the question.

The CS bounds consist of two factors. The first is the norm of the polarization operator, $\|O(\boldsymbol{r})\|$, and the second is the overlap between the orbital densities, $\left|\mu^{2} v^{2}\right|=\int d^{3} r \phi_{i}^{2}(\boldsymbol{r}) \phi_{j}^{2}(\boldsymbol{r})$, where the orbitals $\mu(\boldsymbol{r})$ and $v(\boldsymbol{r})$ are assumed to be Cartesian Gaussians. The square of a Cartesian Gaussian function is bounded by a spherically symmetric Gaussian function with the same total angular momentum $l_{i}=n_{x i}+n_{y i}+n_{z i}$ :

$$
\begin{aligned}
\phi_{i}(\boldsymbol{r})^{2} & =\left(x-x_{i}\right)^{2 n_{x i}}\left(y-y_{i}\right)^{2 n_{y i}}\left(z-z_{i}\right)^{2 n_{z i}} \exp \left(-2 \beta_{i}\left(\boldsymbol{r}-\boldsymbol{r}_{i}\right)^{2}\right) \\
& \leq\left(\boldsymbol{r}-\boldsymbol{r}_{i}\right)^{2 l_{i}} \exp \left(-2 \beta_{i}\left(\boldsymbol{r}-\boldsymbol{r}_{i}\right)^{2}\right)
\end{aligned}
$$

Therefore the overlap of the squared orbitals is bounded by

$$
\begin{aligned}
& \int d^{3} r \phi_{i}^{2}(\boldsymbol{r}) \phi_{j}^{2}(\boldsymbol{r}) \\
\leq & \int d^{3} r r^{2 l_{i}}(\boldsymbol{r}-\boldsymbol{R})^{2 l_{j}} \exp \left(-2 \beta_{i} r^{2}-2 \beta_{j}(\boldsymbol{r}-\boldsymbol{R})^{2}\right),
\end{aligned}
$$

with $\boldsymbol{R}=\boldsymbol{r}_{j}-\boldsymbol{r}_{i}$ the vector pointing from the center of one basis function to the other. This integral is done in spherical coordinates:

$$
\begin{aligned}
\int d^{3} r & \phi_{i}^{2}(\boldsymbol{r}) \phi_{j}^{2}(\boldsymbol{r}) \\
\leq & \int d^{3} r r^{2 l_{i}}\left(r^{2}+R^{2}-2 R r \cos (\theta)\right)^{l_{j}} \\
& \times \exp \left(-2 \beta_{i} r^{2}-2 \beta_{j}\left(r^{2}+R^{2}-2 R r \cos (\theta)\right)\right. \\
= & \left(-\frac{1}{2} \frac{\partial}{\partial \beta_{j}}\right)^{l_{j}} \int_{0}^{2 \pi} d \phi \int_{0}^{\pi} d \theta \sin (\theta) \\
\int_{0}^{\infty} & d r r^{2\left(l_{i}+1\right)} \exp \left(-2 \beta_{i} r^{2}-2 \beta_{j}\left(r^{2}+R^{2}-2 R r \cos (\theta)\right)\right) \\
= & \left(-\frac{1}{2} \frac{\partial}{\partial \beta_{j}}\right)^{l_{j}} 2 \pi \int_{0}^{\infty} d r r^{2 l_{i}+1}\left(-\frac{1}{4 \beta_{j} R}\right) \\
\times & {\left[\exp \left(-2 \beta_{i} r^{2}-2 \beta_{j}(r+R)^{2}\right)-\exp \left(-2 \beta_{i} r^{2}-2 \beta_{j}(r-R)^{2}\right)\right] }
\end{aligned}
$$

For a symmetric integrand, $f(-r)=f(r)$, the integration may be extended over the whole real axis, $\int_{0}^{\infty} d r f(r)=$ $\frac{1}{2} \int_{-\infty}^{\infty} d r f(r)$. After completing the square

$$
\begin{aligned}
& \exp \left(-2 \beta_{i} r^{2}-2 \beta_{j}(r \pm R)^{2}\right)= \\
& \quad \exp \left(-2 \frac{\beta_{i} \beta_{j}}{\beta_{i}+\beta_{j}} R^{2}\right) \exp \left(-2\left(\beta_{i}+\beta_{j}\right)\left(r \pm \frac{\beta_{j}}{\beta_{i}+\beta_{j}} R\right)^{2}\right)
\end{aligned}
$$

and shifting the integration variable by $\pm \frac{\beta_{j}}{\beta_{i}+\beta_{j}}$ the integral becomes

$$
\begin{aligned}
=\left(-\frac{1}{2} \frac{\partial}{\partial \beta_{j}}\right)^{l_{j}} & \left(\frac{-2 \pi}{8 \beta_{j} R}\right) \exp \left(-2 \frac{\beta_{i} \beta_{j}}{\beta_{i}+\beta_{j}} R^{2}\right)\{ \\
& \int_{-\infty}^{\infty} d r\left(r-\frac{\beta_{j}}{\beta_{i}+\beta_{j}} R\right)^{2 l_{i}+1} \exp \left(-2\left(\beta_{i}+\beta_{j}\right) r^{2}\right) \\
& \left.-\int_{-\infty}^{\infty} d r\left(r+\frac{\beta_{j}}{\beta_{i}+\beta_{j}} R\right)^{2 l_{i}+1} \exp \left(-2\left(\beta_{i}+\beta_{j}\right) r^{2}\right)\right\} .
\end{aligned}
$$

Next, the binomial theorem is applied, so that all radial integrals are of the form

$$
\int_{-\infty}^{\infty} d r r^{2 k} \exp \left(-2\left(\beta_{i}+\beta_{j}\right) r^{2}\right)=(2 k-1) ! ! \sqrt{\frac{\pi}{2\left(\beta_{i}+\beta_{j}\right)}}\left(\frac{1}{4\left(\beta_{i}+\beta_{j}\right)}\right)^{k}
$$

After some algebra, the final expression for the upper bound on the overlap between the squares of Cartesian Gaussian orbitals becomes:

$$
\begin{aligned}
& \int d^{3} r \phi_{i}^{2}(\boldsymbol{r}) \phi_{j}^{2}(\boldsymbol{r}) \leq\left(-\frac{\partial}{\partial \beta_{j}}\right)^{l_{j}}(2 \pi)^{3 / 2} \exp \left(-2 \frac{\beta_{i} \beta_{j}}{\beta_{i}+\beta_{j}} R^{2}\right) \\
& \times \sum_{k=0}^{l_{i}} \frac{\left(2 l_{i}+1\right) !}{(2 k) ! !\left(2 l_{i}+1-2 k\right) !} 2^{-2 k-l_{j}-3}\left(\beta_{j} R\right)^{2\left(l_{i}-k\right)}\left(\beta_{i}+\beta_{j}\right)^{-2 l_{i}+k-3 / 2}
\end{aligned}
$$

The partial derivatives with respect to $\beta_{j}$ are taken using the computer algebra package Mathematica. For all combinations of angular momenta $l_{i}, l_{j}=0,1,2,3$ (up to f-functions) C code for the upper bounds is generated automatically. The integral routines were compared with numerical integration on a grid.

The second part of the upper bound is the norm of the polarization operator

$$
\|O(\boldsymbol{r})\|^{2}=\int d^{3} r \frac{x^{2 m_{x}} y^{2 m_{y}} z^{2 m_{z}}}{r^{2 k}}\left(1-\exp \left(-\alpha r^{2}\right)\right)^{2 q}
$$

It is possible to solve this integral analytically; however, for the polarization Hamiltonian in the DRF method only certain values of $k, m_{x}, m y, m_{z}$ and $q$ are possible. The integrals for the relevant combinations of integers as a function of the cutoff exponent $\alpha$ were computed using Mathematica and are listed in table 3 .

For testing, all integrals in Eqn. (109) were evaluated numerically. The centers of the two basis functions were drawn randomly from a ball of radius $5 \AA$, typical of the size of QM 


\begin{tabular}{cccc}
\hline$k$ & $m$ & $q$ & $\|O\|^{2}$ \\
\hline 3 & 1 & 2 & $\frac{8}{3}(1-3 \sqrt{2}+2 \sqrt{3}) \pi^{3 / 2} \alpha^{1 / 2}$ \\
& & & $\frac{128}{75}(-279-30 \sqrt{2}+63 \sqrt{3}+175 \sqrt{5}$ \\
6 & 2 & 4 & $-126 \sqrt{6}+49 \sqrt{7}) \pi^{3 / 2} \alpha^{5 / 2}$ \\
\hline
\end{tabular}

Table 3: Squared norm of polarization operator for typical values of $k, m=m_{x}+m_{y}+m_{z}$ and $q$. Integrals for $\left(m_{x}, m_{y}, m_{z}\right)=(2,0,0),(0,2,0),(0,0,2)$ and $\left(m_{x}, m_{y}, m_{z}\right)=$ $(1,0,0),(0,1,0),(0,0,1)$ differ by a factor of 3 . Since we are interested in an upper bound on all integrals having $m=$ $m_{x}+m_{y}+m_{z}=2$, the larger of the two integrals is shown.

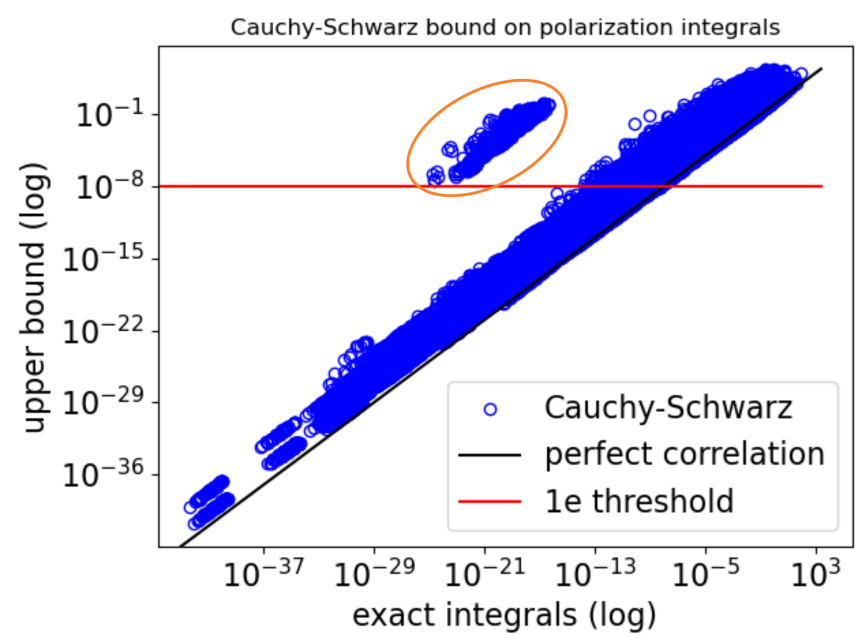

Figure 2: Cauchy-Schwarz bound versus exact polarization integrals for 50 randomly sampled basis function parameters (see text).

regions in $\mathrm{QM} / \mathrm{MM}$ calculations. The polarizable atom was always placed at the origin. The exponents of the basis functions were also drawn randomly from the interval [0.01,2.01]. In total, parameters for 50 pairs of shells are generated. For a pair of centers, all combinations of angular momenta up to d-functions were enumerated. Figure 2 shows a scatter plot of the upper bounds versus the exact polarization integrals. The diagonal straight black line indicates a hypothetical perfect bound.

All points in Figure 2 lie above the diagonal black line, so the bound is rigorous. Furthermore, for most integrals, the bound is within seven orders of magnitude, which is typical for CS bounds involving Cartesian Gaussian basis functions. If the threshold for neglecting one-electron integrals is set to $10^{-8}$ Hartree (red horizontal line in Fig. 2), the CS bound shows that most integrals can be neglected. We note, however, that for some small integrals the bound exceeds the true integral by more than seven orders of magnitude, shown within the gold circle in Fig. 2. These can be understood as arising from symmetry. For some combinations of angular components, the integrals are numerically zero by symmetry, but they are still included since the screening is applied to blocks of basis functions with the same total angular momentum. The number of these integrals is relatively small, however, so including them does not incur a large computational effort.

\section{Usage of the Integral Library}

The integral routines for all cases are made available as a library [19] written in C++/CUDA. The library contains separate implementations specialized for CPU and GPU architectures. Python bindings give access to the underlying $\mathrm{C}++$ classes and wrapper functions around the CUDA kernels.

CPU implementation: The snippet of python code below illustrates how to use the CPU version. Basis functions on an atomic center $\left(x_{i}, y_{i}, z_{i}\right)$ with the orbital exponent $\beta_{i}$ are grouped into shells of total angular momentum $l_{i}=n_{x i}+n_{y i}+n_{z i}$. Integrals for all angular momentum components of a shell are processed in one go so that intermediate results can be reused. The library defines the class PolarizationIntegral for evaluating individual integrals between shells. First, an integral object has to be created. Its constructor takes the data for the bra and ket shells as well as the parameters of the polarization operator as input:

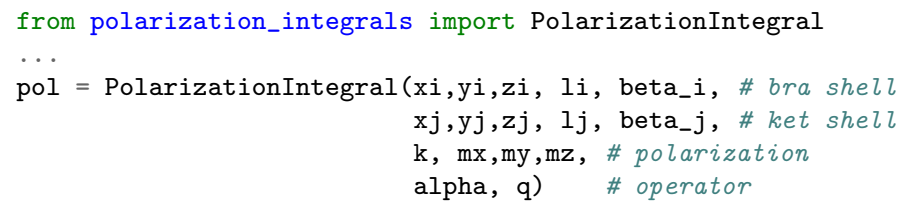

The member function compute_pair (...) of the integral object can then be called repeatedly to iterate over all combinations of angular components $\left(n_{x i}, n_{y i}, n_{z i}\right)$ and $\left(n_{x j}, n_{y j}, n_{z j}\right)$ belonging to the shells with total angular momenta $l_{i}$ and $l_{j}$ :

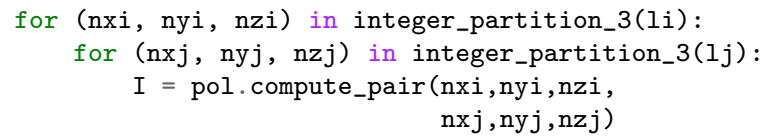

The function integer_partition_3(1) is assumed to return a list of all possible integer partitions $\left(n_{x}, n_{y}, n_{z}\right)$ of size 3 of the integer $l$ such that $n_{x}+n_{y}+n_{z}=l$.

GPU implementation: The GPU implementation makes heavy use of $\mathrm{C}++$ templates. The template parameters are the precision (float or double) as well as the integer parameters $\mathrm{k}, \mathrm{mx}, \mathrm{my}, \mathrm{mz}$ and the cutoff power $\mathrm{q}$ defining the type of the polarization operator. Being a real number, the cutoff exponent $\alpha$ cannot be used as a template parameter and has to be passed as a function argument. Optimized instances of the templated functions are compiled for each combination of the template parameters.

The computation of polarization integrals proceeds in two steps. First, an array of all pairs of basis functions has to be generated. The upper bound may be used to skip negligible pairs. Each pair (instance of class PrimitivePair) contains the information about the position, total angular momentum, contraction coefficient and orbital exponent of the bra and ket primitive shells (both instances of the Primitive class). In the second step, the pair list is transferred to the device memory, and the kernel function 


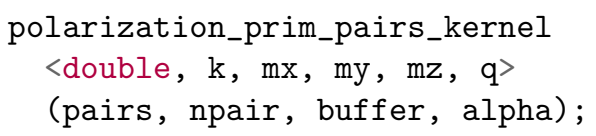

processes all npair pairs in parallel putting the output into a buffer array. Since each primitive shell with angular momentum $l$ contains $N_{L}(l)=(l+1)(l+2) / 2$ Cartesian angular momentum components (all combinations of powers $n_{i}$ such that $n_{x}+n_{y}+n_{z}=l$ ), each pair of primitive shells $i$ and $j$ generates an integral block of size $N_{\mathrm{L}}\left(l_{i}\right) \times N_{\mathrm{L}}\left(l_{j}\right)$ in the output buffer.

For more information refer to the source code of the test cases and examples.

\section{Acknowledgments}

This work was supported by the National Natural Science Foundation of China Research Fund for International Excellent Young Scientists (Grant No. 22150610466), the National Natural Science Foundation of China (Grant No. 22173060), the Ministry of Science and Technology of the People's Republic of China National Foreign Experts Program Fund (Grant No. QN2021013001L), start-up funds from NYU Shanghai, and a pre-tenure grant from the NYU-ECNU Center for Computational Chemistry. We thank Peter Schwerdtfeger for a helpful discussion.

\section{Appendix A. Analytical Gradients}

Analytical gradients of the polarization integrals in Eqn. (2) with respect to the centers of the Gaussian-type orbitals are obtained in analogy to gradients of overlap integrals. We exploit the fact that the first derivative of a Cartesian Gaussian function with respect to the center is the sum of two Gaussian functions with different powers:

$$
\begin{aligned}
\frac{d}{d x_{i}}\left(x-x_{i}\right)^{n_{x i}} e^{-\beta_{i}\left(x-x_{i}\right)^{2}}= & -n_{x i}\left(x-x_{i}\right)^{n-1} e^{-\beta_{i}\left(x-x_{i}\right)^{2}} \\
& +2 \beta_{i}\left(x-x_{i}\right)^{n+1} e^{-\beta_{i}\left(x-x_{i}\right)^{2}} .
\end{aligned}
$$

If the dependence of the polarization integral on the Cartesian powers of orbitals $i$ and $j$ is denoted by $I\left(n_{x i}, n_{y i}, n_{z i} ; n_{x j}, n_{y j}, n_{z j}\right)$, the derivative with respect to the $x$ coordinate of orbital $i$ can be expressed as

$$
\frac{d}{d x_{i}} I\left(n_{x i}, \ldots\right)=-n_{x i} I\left(n_{x i}-1, \ldots\right)+2 \beta_{i} I\left(n_{x i}+1, \ldots\right)
$$

and similarly for the other coordinates of centers $i$ and $j$. The polarization integrals are three-center integrals that only depend on the relative vectors between the centers. Therefore gradients with respect to the position of the polarization operator $\boldsymbol{r}_{O}$ (taken as the origin) can be assembled easily from the gradients with respect to the bra and ket centers:

$$
\nabla_{\boldsymbol{r}_{O}} I=-\nabla_{\boldsymbol{r}_{i}} I-\nabla_{\boldsymbol{r}_{j}} I
$$

\section{References}

[1] P. Fuentealba, H. Preuss, H. Stoll, L. Szentpály, A proper account of corepolarization with pseudopotentials: single valence-electron alkali compounds, Chem. Phys. Lett. 89 (5) (1982) 418-422.

[2] W. Müller, J. Flesch, W. Meyer, Treatment of intershell correlation effects in ab initio calculations by use of core polarization potentials. method and application to alkali and alkaline earth atoms, J. Chem. Phys. 80 (7) (1984) 3297-3310.

[3] B. T. Thole, P. T. van Duijnen, On the quantum mechanical treatment of solvent effects, Theor. Chim. Acta 55 (4) (1980) 307-318.

[4] B. T. Thole, P. T. Van Duijnen, The direct reaction field hamiltonian: Analysis of the dispersion term and application to the water dimer, Chem. Phys. 71 (2) (1982) 211-220.

[5] A. H. De Vries, P. T. Van Duijnen, Solvatochromism of the $\pi^{*} \leftarrow \mathrm{n}$ transition of acetone by combined quantum mechanical—classical mechanical calculations, Int. J. Quantum Chem. 57 (6) (1996) 1067-1076.

[6] A. De Vries, P. T. Van Duijnen, A. Juffer, J. Rullmann, J. Dijkman, H. Merenga, B. T. Thole, Implementation of reaction field methods in quantum chemistry computer codes, J. Comput. Phys. 16 (1) (1995) 3755.

[7] H. Stoll, P. Fuentealba, P. Schwerdtfeger, J. Flad, L. v. Szentpály, $\mathrm{H}$. Preuss, $\mathrm{Cu}$ and $\mathrm{Ag}$ as one-valence-electron atoms: $\mathrm{CI}$ results and quadrupole corrections for $\mathrm{Cu}_{2}, \mathrm{Ag}_{2}, \mathrm{CuH}$, and $\mathrm{AgH}$, J. Chem. Phys. 81 (6) (1984) 2732-2736. doi:10.1063/1.447992.

[8] P. Schwerdtfeger, H. Silberbach, Multicenter integrals over long-range operators using cartesian gaussian functions, Phys. Rev. A 37 (8) (1988) 2834.

[9] P. Schwerdtfeger, H. Silberbach, Erratum: Multicenter integrals over long-range operators using cartesian gaussian functions, Phys. Rev. A 42 (1) (1990) 665.

[10] M. J. Smit, Multicenter integrals over polarization potential operators, Int. J. Quantum Chem. 73 (5) (1999) 403-416.

[11] L. E. McMurchie, E. R. Davidson, One-and two-electron integrals over cartesian gaussian functions, J. Comput. Phys. 26 (2) (1978) 218-231.

[12] B. Gao, A. J. Thorvaldsen, K. Ruud, Gen1int: A unified procedure for the evaluation of one-electron integrals over gaussian basis functions and their geometric derivatives, Int. J. Quantum Chem. 111 (4) (2011) 858872.

[13] E. F. Valeev, Libint: A library for the evaluation of molecular integrals of many-body operators over gaussian functions, http://libint.valeyev.net/, version 2.7.1 (2021).

[14] P. Schwerdtfeger, H. Silberbach, Erratum: Multicenter integrals over long-range operators using cartesian gaussian functions [phys. rev. a 37, 2834 (1988)], Phys. Rev. A 103 (2021) 069901. doi:10.1103/PhysRevA.103.069901.

URL https://link.aps.org/doi/10.1103/PhysRevA.103.069901

[15] W. R. Inc., Mathematica, Version 11.0.0, champaign, IL, 2021. URL https : //www. wolfram. com/mathematica

[16] A. D. Becke, A multicenter numerical integration scheme for polyatomic molecules, J. Chem. Phys. 88 (4) (1988) 2547-2553.

[17] library for numerical integration on multicenter becke grids, https://github.com/humeniuka/becke_multicenter_integration.

[18] S. G. Johnson, Faddeeva package. URL http: //ab-initio.mit.edu/Faddeeva

[19] integral library for polarization integrals (c++ and python), https://github.com/humeniuka/polarization_integrals. 